\title{
Research on the Principle of Residual Current Protection Technology Based on Transient Waveform Criterion
}

\author{
Xiao Guolei ${ }^{1}$, Song Yan $^{1}$, Ye Juwei ${ }^{1}$, Lv Chengcong ${ }^{1 *}$, Wu Binfeng ${ }^{1}$, Jiang Feng ${ }^{2}$, Jin \\ Weidong $^{2}$ \\ ${ }^{1}$ Lishui Power Supply Company, State Grid Zhejiang Electric Power Co. Ltd., Lishui 323000, \\ Zhejiang Province, China; \\ ${ }^{2}$ Lishui Power Industry Group Co.,Ltd, Lishui, 323000,China
}

\begin{abstract}
The paper uses a magnetic modulation current transformer method to detect transient waveform residual currents containing pulsating DC components and even smooth DC components. At the same time, it uses IIR-based digital low-pass filters to filter the collected signals, which improves Filtering accuracy. The software design is based on the floatingpoint DSP-TMS320F28335, which realizes the accurate sampling and analysis of the transient waveform residual current, and the transient waveform residual current detection meets the standard requirements.
\end{abstract}

Keywords: Transient waveform, residual current protection; low-pass filter.

\section{Introduction}

With the increase in the number and types of electrical equipment, the number and forms of equipment leakage faults continue to increase. The waveform of the transient residual current signal not only contains power frequency sinusoidal $\mathrm{AC}$, but also contains pulsating DC components and even smooth DC components. According to the different waveform characteristics of the residual current, the International Electrotechnical Commission IEC divides the residual current protector into four types: AC type, A type, F type and B type. Various types of residual current protection appliances can only act on the corresponding residual current: AC type residual current protection appliances can only operate correctly when AC residual current; Type A can operate correctly on $\mathrm{AC}$ residual current and pulsating DC residual current; Type F can operate correctly on both AC residual current and pulsating DC residual current and can detect $1000 \mathrm{~Hz}$ leakage signal at the same time; Type B can not only operate correctly for AC residual current and pulsating DC residual current, but also for smooth DC residual current. Therefore, Type B is also known as a full current sensitive residual current protection appliance.

As the main equipment to protect the safe operation of the rural low-voltage power grid,

\footnotetext{
*663597309@qq.com
} 
the residual current-operated protection device based on the transient waveform criterion plays a vital role in the safe and stable operation of the rural low-voltage power grid. The transient waveform as the criterion refers to that the current flowing through the human body is a non-sinusoidal, non-linear current waveform. Human body contact current in a short time (about 2 to 3 cycles), the skin impedance is a time-varying network, its resistance value changes from large to small, and later transformed into a time-invariant network. This characteristic of skin impedance determines that the contact current flowing through the human body is an increasing periodic function during the initial period of time [1]. In addition to transient waveform protection, the residual current operated protector based on the transient waveform criterion will also have multiple protection functions such as automatic tracking, overcurrent, and residual current. It can be designed as an integrated type, multiple functions, and small size. Intelligent, the main circuit current can be set leakage circuit breaker.

\section{Related theoretical basis}

\subsection{Residual current detection method}

\subsubsection{Electromagnetic current transformer method.}

Power frequency AC residual current, when the line is working normally, according to Kirchhoff's current law, due to the balance of the three-phase current, the sum of the current vectors passing through the primary side of the residual current transformer is zero, namely:

$$
I_{1}+I_{2}+I_{3}=0
$$

At this time, the sum of the magnetic flux in the electromagnetic current transformer is zero, that is:

$$
\Phi_{1}+\Phi_{2}+\Phi_{3}+\Phi_{n}=0
$$

In the event of personal electric shock or other leakage faults (single-phase grounding, two-phase short circuit, etc.), the three-phase current balance is destroyed, and the primary current vector sum $I_{\Delta}$ of the residual current transformer is no longer zero. We call $I_{\Delta}$ the residual current (also Called leakage current), namely:

$$
I_{1}+I_{2}+I_{3}+I_{n}=I_{\Delta}
$$

At this time, the flux vector sum $\Phi_{\Delta}$ of the residual current transformer is no longer zero:

$$
\Phi_{1}+\Phi_{2}+\Phi_{3}+\Phi_{n}=\Phi_{\Delta}
$$

Under the action of the alternating magnetic flux, the induced electromotive force E2 is generated on the secondary side of the transformer [2]. This signal enters the single-chip microcomputer through the sampling resistor and the signal conditioning circuit to perform the leakage protection function after being sampled and processed. 


\subsubsection{Hall current sensor method.}

There is a magnetic field perpendicular to the Hall element or a magnetic field of the normal phase component of the Hall element. When a current is applied to the Hall element, under the combined action of the magnetic field and the current, the Hall element will generate the Hall potential $E_{H}, E_{H}$ which is combined with the current and magnetic field. The intensity is proportional, namely:

$$
E_{H}=K_{H} * I * B
$$

$K_{H}$ is the sensitivity coefficient of the Hall element, which is related to the material and shape characteristics of the Hall element. According to different working principles, Hall current sensors are divided into direct measurement type Hall current sensors and magnetic balance type Hall current sensors, both of which are composed of primary circuit, soft magnetic material concentrating ring, Hall element, amplifier circuit, etc. composition [3].

\subsection{Working principle of direct measurement Hall current sensor}

The magnetic ring of soft magnetic material gathers the magnetic field generated by the primary current to the Hall element. The Hall element generates current through the DC voltage power supply. Under the action of the magnetic field generated by the current and the detection current, the Hall electromotive force is generated. Because the general detection current is small, the Hall electromotive force generated is small, and then the output is amplified by the differential amplifier circuit, as shown in Figure 1.

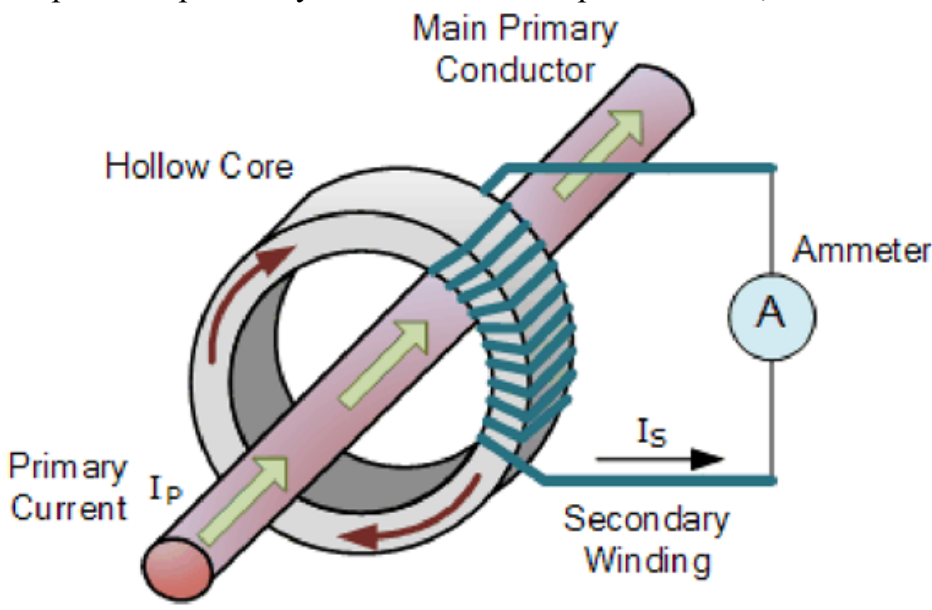

Construction

\section{Main Primary Conductor}

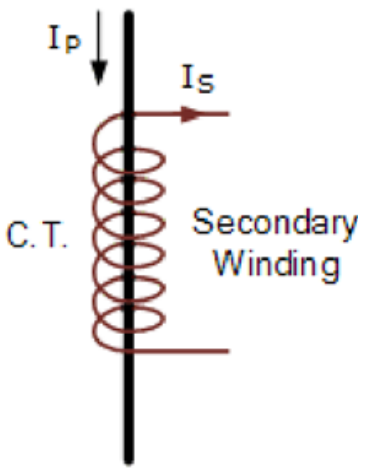

Circuit

Symbol

Figure 1. Schematic diagram of direct current sensor

The working principle of the magnetic balance Hall current sensor is: the primary current $I_{p}$ passes through the magnetic concentrating ring. The magnetic concentrating ring generates a magnetic field due to electromagnetic induction. The magnetic field component perpendicular to the Hall element causes the Hall element to generate Hall electromotive force $E_{H}$; The electromotive force $E_{H}$ is amplified by the amplifier circuit, and then through the power amplifier circuit to turn on the power tube to generate a compensation current $I_{s}$; this current $I_{s}$ is wound around the secondary coil of the magnetization ring 
through multiple turns, and the secondary coil makes the magnetization ring generate a compensation electromotive force $E_{s}$; The direction of the compensation electromotive force $E_{s}$ is exactly opposite to the Hall electromotive force $E_{H}$. When the compensation electromotive force $E_{s}$ is exactly equal to the Hall electromotive force $E_{H}$, until the Hall element maintains the working state of zero flux, the feedback ends. Figure 2 shows the principle of a magnetic balance current sensor.

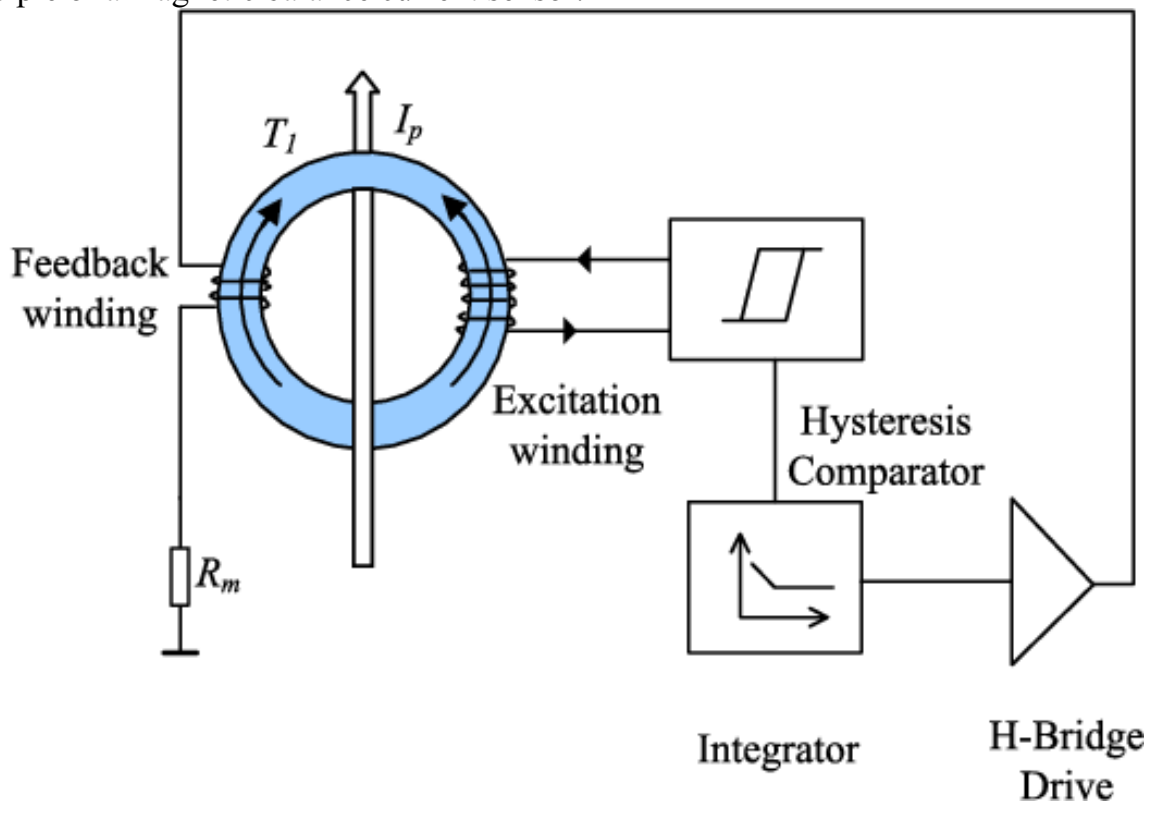

Figure 2. Principle of magnetic balance current sensor

\subsection{Digital sampling filter algorithm}

The arithmetic mean filtering algorithm is to find a value $\mathrm{Y}$ that minimizes the sum of squared deviations of each sampling point value as the sampling value. Let $\mathrm{E}$ be the sum of squared deviations between $\mathrm{Y}$ and each sampling point value $x_{i}, i=1,2,3, \ldots, N$, namely

$$
E=\sum_{i=1}^{N}\left(Y-x_{i}\right)^{2}
$$

By solving the minimum value of $\mathrm{E}$

$$
k=n^{2}[0.417+3.14 \log (O D / I D)]
$$

When the measured signal that changes slowly contains random interference signals, it is suitable to use the arithmetic average method, such as temperature and humidity. However, since this filtering method must continuously collect $\mathrm{N}$ points of data each time it is sampled, it is not suitable for occasions where the analogy signal changes quickly or requires high real-time performance [4]. 


\section{Technical principle realization}

\subsection{Hardware implementation}

The transient waveform RCD mainly includes transformer, signal conditioning circuit, microprocessor, excitation voltage frequency detection circuit, action execution circuit, residual current display circuit and other parts, as shown in Figure 3.

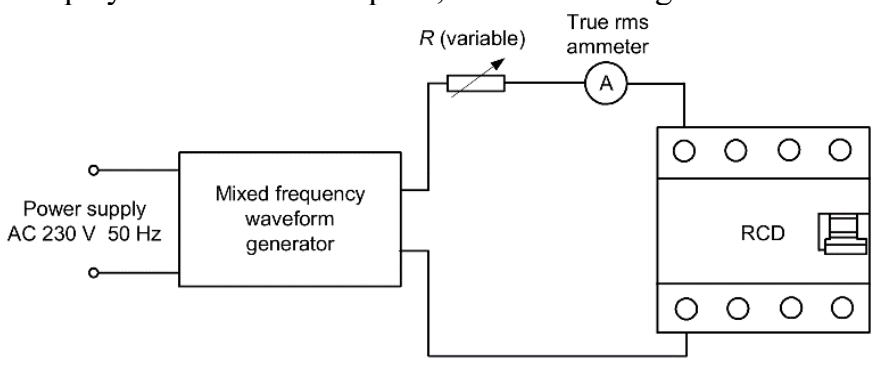

Figure 3. Hardware structure block diagram

\subsubsection{Detection part.}

This article uses a single-core open-loop magnetic modulation AC/DC toroidal sensitive toroidal current transformer (AC/DC-RCT). The magnetic modulation AC/DCRCT is mainly composed of toroidal core, sampling resistor Rs, differential operational amplifier, and $\mathrm{H}$-bridge buffer chip. Among them, the protected power line passes through the transformer core, and a secondary winding is wound on the core, the number of turns is N2; the $\mathrm{H}$-bridge buffer chip outputs positive and negative symmetrical square wave excitation voltage $\mathrm{U}(\mathrm{t})$ applied to the secondary The winding forms a series loop with the sampling resistor.

\subsubsection{Power supply section.}

This design uses a transformer to simulate the power supply circuit, as shown in Figure 4. It can be seen from Figure 4 that the power supply directly fetches single-phase power from the power grid, and steps down through electrolytic capacitors $\mathrm{C} 7, \mathrm{C} 8$, common mode inductors, and power frequency transformers. The diode 1N4007 performs bridge rectification to generate $+12 \mathrm{~V}$ DC. It can be seen from Figure 4 that the $+12 \mathrm{~V}$ direct current passes through the LM2596 integrated circuit chip to generate a stable $+5 \mathrm{~V}$ voltage to power the single-chip microcomputer and other chips. The LM2596 switching voltage regulator step-down power management monolithic integrated circuit can output $3 \mathrm{~A}$ drive current and has a good Linearity and load regulation characteristics, using the internal oscillation frequency of $150 \mathrm{kHz}$, low power and high efficiency [5]. 


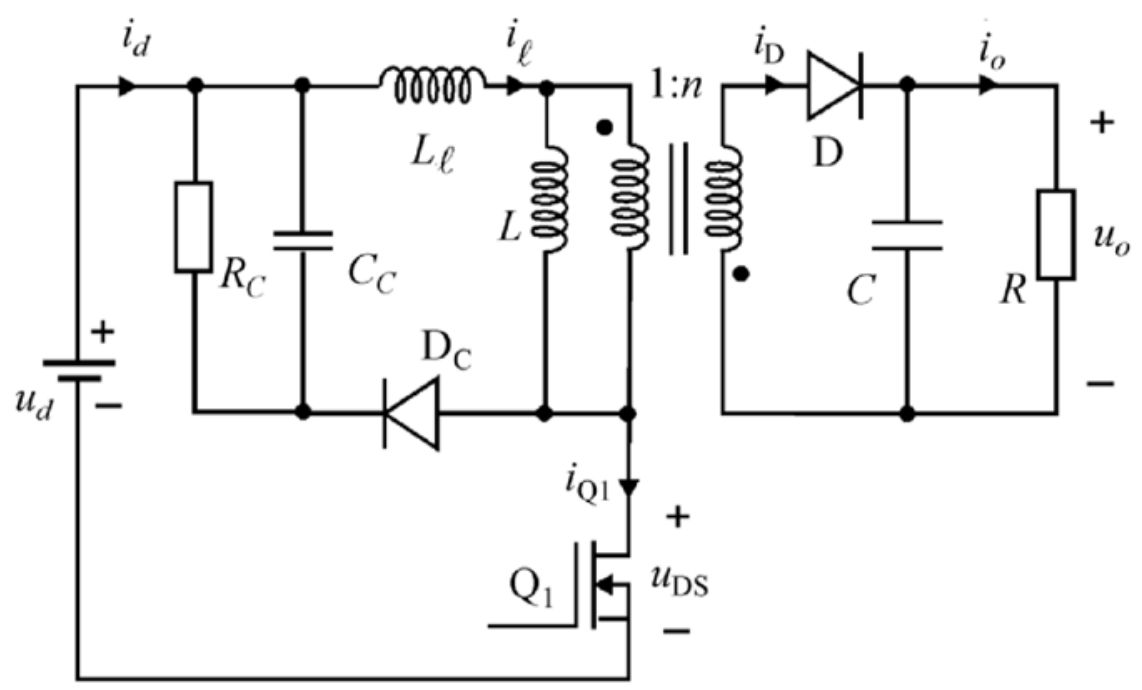

Figure 4. Power circuit

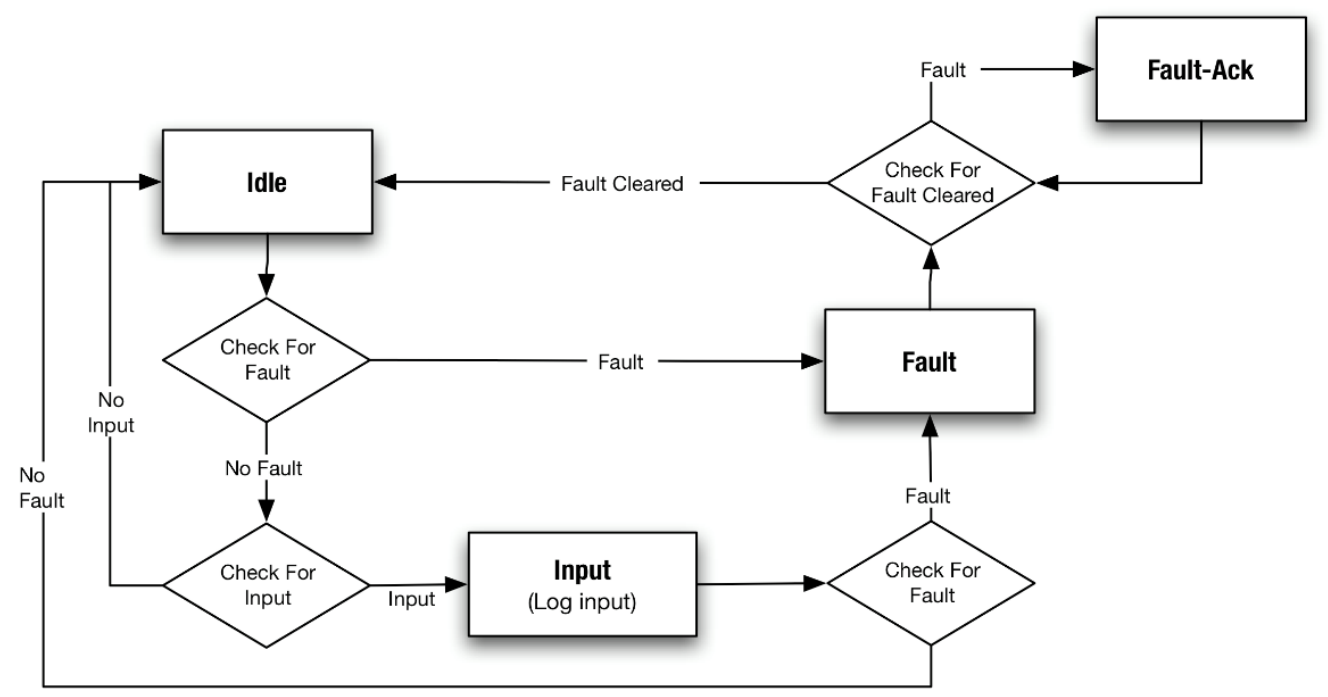

Figure 5. Software process

\subsection{Software design}

The software process is shown in Figure 5. Initialize first, receive the sampling start trigger signal DSP and start sampling, sampling 1024 points per cycle, the sampling period is $0.03125 \mathrm{~ms}$, the sampling window time is $1024 \times 0.03125=32 \mathrm{~ms}$, the sampling point enters the IIR digital filter Subroutine, filter processing. In order to ensure the protection of the residual current under different waveforms, a waveform recognition subroutine was written to divide the residual current waveform into 5 waveforms: $\mathrm{DC}$, sine $\mathrm{AC}$, half wave, $90^{\circ}$ wave and $135^{\circ}$ wave. According to different waveforms, the conversion between average value and effective value is performed to keep the characteristics of residual current protection consistent. According to the residual current waveform, the DSP converts the average value to an effective value and compares it with the set action value. If it is greater 
than the action value, the counter value increases; then, compares the count value $\mathrm{N}$ with the set threshold value, if $\mathrm{N}$ is greater than Set the threshold to execute the trip procedure, otherwise it will enter the next cycle.

\section{Experimental test}

Test the transient waveform RCD prototype. During the test, apply a test current with the same amplitude but different frequency and waveform to the magnetic modulation RCD, and record the test results of the prototype action value. According to the programming, the transient current sampling values of different fault locations and fault forms are analysed, and the results are shown in Table 1.

Table 1. The wavelet transforms analysis results of the fault when the voltage exceeds $0^{\circ}$

\begin{tabular}{|c|c|c|c|c|c|c|}
\hline parameter & $K_{1}$ & $K_{2}$ & $K_{3}$ & $K_{4}$ & $K_{5}$ & $K_{6}$ \\
\hline Ratio (Single-phase grounded phase A) & 245.7922 & 127.5801 & 89.1720 & 96.7381 & 29.9232 & 0.0014 \\
\hline Ratio (two-phase short circuit BC) & 350.8100 & 96.7300 & 59.2500 & 36.0108 & 30.7453 & 0.0002 \\
\hline Ratio (three-phase short circuit) & 342.6500 & 96.2600 & 36.2700 & 36.0300 & 29.9200 & 0.0004 \\
\hline TMH (Single-phase grounded phase A) & 0.0000 & 0.0005 & 0.0009 & 0.0009 & 0.0009 & 0.0000 \\
\hline TMH (two-phase short circuit BC) & 0.0000 & 0.0005 & 0.0009 & 0.0009 & 0.0009 & 0.0000 \\
\hline TMH (three-phase short circuit) & 0.0000 & 0.0005 & 0.0009 & 0.0009 & 0.0009 & 0.0000 \\
\hline
\end{tabular}

Analysis of Table 1 shows that: 1) The new criterion can be used to clearly distinguish whether the fault point is within the setting range, that is, for the fault that the modulus maximum high-frequency component occurs after $0.9 \mathrm{~ms}$, the Ratio and Ratiol are both small. And the difference in ratio is very significant. 2) The fault at the end of the protection zone (K4), compared with the fault at the exit of the adjacent line (K5), for some faults, the changes in Ratio and Ratiol are not obvious [6]. However, the Ratio and Ratio1 values that can satisfy the faults in the area are larger than those outside the area. If the value of Ratio and Ratiol at K5 is used for the setting calculation, the value of the reliability coefficient must be very close to 1 , so it can only satisfy the theoretical setting of transient protection under the form of single-phase ground fault. The selectivity of phaseto-phase faults needs further study.

\section{Conclusion}

It can be seen from the test results that the transient waveform RCD of this design has high accuracy for sinusoidal AC, half-wave and DC waveforms, and relatively poor accuracy for $90^{\circ}$ and $135^{\circ}$ waves, but it can basically meet the requirements of the standard and has certain practicality. Sex. Digital filtering based on DSP improves filtering accuracy and simplifies hardware design, but at the same time increases costs. Due to the principle of magnetic modulation, the magnetic core needs to be in a state of oversaturation, and the power consumption is large, so it needs to be improved.

\section{Acknowledgments}

This work was financially supported by: Collective Enterprise Science and Technology Project of State Grid Zhejiang Electric Power Co., LTD. (Project No.: LSJTKJ-2019-6). 


\section{References}

1. Czapp, S. Comparison of residual current devices tripping characteristics for selected residual current waveforms. Elektronika Ir Elektrotechnika, 100(4) (2015) 7-10.

2. Han, X., Sheng, W., Du, S., Su, J., \& Liu, G. Novel protection scheme for residual current device-based electric fault time detection and touch current identification. Iet Generation Transmission \& Distribution, 11(10) (2017) 2478-2488.

3. Weng, H., Wang, S., Lin, X., Li, Z., \& Huang, J. A novel criterion applicable to transformer differential protection based on waveform sinusoidal similarity identification. International Journal of Electrical Power \& Energy Systems, 105(2) (2019) 305-314.

4. Grandin, R., Doin, M. P., Bollinger, L., Pinel-Puyssegur, B., Ducret, G., \& Jolivet, R., et al. Long-term growth of the himalaya inferred from interseismic insar measurement. Geology, 40(12) (2017) 1059-1062.

5. Yair, P., Simon, V. G., De, L. F. P., Lamme, V. A. F., \& Seth, A. K. Expectations accelerate entry of visual stimuli into awareness. Journal of Vision, 15(8) (2015) 13-19.

6. Ming, T., Wu, Y., Peng, C., \& Tao, Y. Thermal analysis on a segmented thermoelectric generator. Energy, 80(2) (2015) 388-399. 